\title{
Wall friction and effective viscosity of a homogeneous dispersed liquid- liquid flow in a horizontal pipe
}

\author{
A. Pouplin \\ CNRS; Fédération de recherche FERMaT FR 3089, 31432 Toulouse, France \\ IFP, Dept. de Mécanique Appliquée, 92852 Rueil-Malmaison, France \\ Université de Toulouse, INPT - UPS; Laboratoire de Génie Chimique, 31432 Toulouse, France

\section{O. Masbernat} \\ CNRS; Fédération de recherche FERMaT FR 3089, 31432 Toulouse, France \\ Université de Toulouse, INPT - UPS; Laboratoire de Génie Chimique, 31432 Toulouse, France
}

\section{S. Décarre}

IFP, Dept. de Mécanique Appliquée, 92852 Rueil-Malmaison, France

\author{
A. Liné \\ CNRS; Fédération de recherche FERMaT FR 3089, 31432 Toulouse, France \\ Université de Toulouse, INSA; Laboratoire d'Ingénierie des Systèmes Biologiques et des Procédés, F-31077 \\ Toulouse Cedex 4, France
}

\begin{abstract}
Homogenous oil in water dispersion has been investigated in a horizontal pipe. The mean droplet size is $25 \mu \mathrm{m}$. Experiments were carried out in a $7.5 \mathrm{~m}$ long transparent pipe of $50 \mathrm{~mm}$ internal diameter. The wall friction has been measured and modelled for a wide range of flow parameters, mixture velocities ranging from 0.28 to $1.2 \mathrm{~m} / \mathrm{s}$ and dispersed phase volume fractions up to 0.6 , including turbulent, intermediate and laminar regimes. Flow regimes have been identified from velocity profiles measured by PIV in a matched refractive index medium. It is shown that the concept of effective viscosity is relevant to scale the friction at the wall of the dispersed flow. Based on mixture properties, the friction factor follows the Hagen-Poiseuille and the Blasius' law in laminar and turbulent regimes respectively. Interestingly, the transition towards turbulence is delayed as the dispersed phase fraction is increased.
\end{abstract}

Keywords: liquid-liquid pipe flow, homogeneous dispersed flow, PIV, effective viscosity, wall friction

\section{Introduction}

In this article, the determination of the wall friction law of a homogeneous dispersed liquid-liquid flow in a horizontal pipe is addressed. This problem is of particular interest in oil extraction processes and in many other industries dealing with the transport of emulsions. ${ }^{1}$ Oil production is still associated with a high water throughput which tends to increase as wells are aging. There have been a number of experimental studies dealing with the flow configurations of co-current liquid-liquid flow in horizontal pipes. Depending on the mixture velocities and phase ratios, the flow can take different spatial configurations, dispersed or stratified, or dual layer stratified-dispersed. ${ }^{2-5}$ Fully dispersed flows occur at high mixture velocities, when the 
turbulence level is high enough to maintain a suspension of drops in the bulk flow. The flow is said to be homogeneous when the drops are uniformly distributed across the whole pipe cross-section.

Homogeneous dispersed flows have given rise to quite a number of experimental studies aiming at measuring and scaling local flow field in gas-liquid, solid-liquid and gasparticle dispersed turbulent channel flows. ${ }^{6-12}$ Most of them deal with inertial particles or high particle Reynolds numbers (for bubbles). Detailed measurements in turbulent liquid-liquid pipe flows have been poorly addressed. ${ }^{13,14}$

Modelling homogeneous dispersed flows cannot be undertaken without addressing the question of turbulence damping or enhancement. An informative review of this problem can be found in the paper of Sundaresan et al., ${ }^{15}$ highlighting the lack of available theory to predict such mechanisms, even for dilute conditions, simple geometries and small size particles (i.e smaller or of the order of the Kolmogorov scale). Depending on the particle size with respect to turbulent length scales, the Stokes number (the ratio between the inclusion relaxation time and the fluid time scale "seen" by the inclusion), and the particle Reynolds number, the turbulence can be enhanced or reduced. The inclusion of the effect of concentration and polydispersity has not yet been achieved so far.

In the case of low inertia suspensions in liquids, (i.e low Stokes and particle Reynolds numbers), the mean slip velocity and particle inertia are negligible and it is generally accepted that the fluid-particle interaction can be described through an effective viscosity effect which increases with increasing the concentration of the dispersed phase. This assertion is mainly based upon wall friction measurements in pipe flow as a function of drop concentration. ${ }^{16,17}$ $\mathrm{Pal}^{18}$ observed a drag reduction effect with unstable dispersions, which disappears or is significantly reduced in the case of surfactant stabilized emulsions. Pal also found that stabilized emulsions can be described as a single phase flow with effective properties in both laminar and turbulent regimes. Similar results were also observed in vertical pipe flow. ${ }^{17,19,20}$

An interesting problem is the laminar-turbulent regime transition in dispersed flows. In the case of fine emulsions, the results of $\mathrm{Pal}^{18}$ show that Hagen-Poiseuille law is valid for mixture Reynolds numbers ranging up to 4000, suggesting that the drops tend to delay the transition to turbulence. More recently, Matas et al. ${ }^{21}$ studied this problem with neutrally buoyant solid particles in a pipe flow. Their results clearly show that the transition towards turbulence occurs at smaller mixture Reynolds numbers for large particle-to-pipe diameter ratios, while it is unchanged for smaller particles at concentrations lower than 0.2-0.25. Above this concentration, small particles delay the transition to turbulence. Therefore, it can be 
concluded that even with low inertia suspensions where effective properties can be used to scale the wall friction, laminar-turbulent transition is a function of not only the mixture Reynolds number, but also of the concentration. The last observation also underlines the need for detailed flow measurements in concentrated suspension flows.

In the present work, the hydrodynamic local field of a homogeneous liquid-liquid dispersion flowing in a horizontal pipe has been measured with Particle Image Velocimetry, using the refractive index matching technique. Combining these data with pressure drop measurements, the wall friction and effective viscosity of the emulsion have been identified and scaled in a wide range of flow parameters (mixture velocity and drop concentration), from the turbulent to the laminar regime.

This article is structured as follows: In the first section, the liquid phase system, the experimental device, the measurement technique and the flow parameters are presented. The homogeneous flow domain is drawn on a mixture velocity-concentration map and the stability of the dispersion is also addressed. The results are then presented and discussed for the three different flow regimes studied: turbulent, laminar and intermediate. For each flow regime, the longitudinal velocity profile and the pressure drop measurement are presented and discussed at different concentrations and velocities. The effective viscosity derived from these measurements is then discussed and its evolution with concentration is compared to existing models. In the last section, the wall friction factor is presented and discussed as a function of the mixture Reynolds number. The main results are summarized in the Conclusion section.

\section{Experimental}

The experimental device and fluids used in this study are similar to those of Conan et al. $^{22}$ who studied the stratified-dispersed flow configuration. The experimental loop was modified in order to generate homogeneous flow of small drops using the centrifugal pump of the continuous (aqueous) loop.

\section{Phase system}

The properties of the two fluids are reported in Table 1. The continuous phase is an aqueous solution of glycerin at $43 \% \mathrm{vol} / \mathrm{vol}$. The dispersed phase is $\mathrm{n}$-heptane (technical grade), immiscible with water. At $29^{\circ} \mathrm{C}$, the two phases have their refractive index matched (1.385) allowing light transmission without deviation. 
Table 1: Physical properties of the fluids at $29^{\circ} \mathrm{C}$

\begin{tabular}{lccccc} 
Phases & Density & Viscosity & Refractive Index & Interfacial tension \\
& & $\left(\mathrm{kg} \cdot \mathrm{m}^{-3}\right)$ & $($ Pa.s $)$ & $(-)$ & $\left(\mathrm{N} . \mathrm{m}^{-1}\right)$ \\
\hline Dispersed & Heptane & 684 & $410^{-4}$ & 1.385 & 0.031 \\
Continuous & Water-Glycerin (43\% vol) & 1102 & $3.210^{-3}$ & 1.385 &
\end{tabular}

\section{Experimental set-up}

A schematic of the whole setup is shown in Figure 1. The device comprises a $7.5 \mathrm{~m}$ long horizontal pipe of $50 \mathrm{~mm}$ internal diameter, a gravity settler, an entry section, two centrifugal pumps, and a secondary loop with heat exchangers which regulate the flow temperature in the loop. In order to implement optical techniques, the duct is made of poly methyl-methacrylate (PMMA), and movable square boxes (30 $\mathrm{cm}$ long) filled with water are mounted along the pipe to reduce optical distortions.

The two fluids are stored in the gravity settler (400L). Each phase is pumped from the settler to the pipe inlet by centrifugal pumps of variable rotation speed. Flowrates are measured with the help of an electromagnetic flowmeter and a vortex flowmeter, for the aqueous and the organic phase respectively, with an accuracy of $0.5 \%$. Oil and water phases merge in a Y-junction, at the pipe inlet. Upstream of the Y-junction a convergent section has been mounted on the water loop to eliminate or reduce secondary flows in the pipe. The startup procedure of the experimental device is operated in two stages. At first, each phase is continuously fed in the pipe and separated in the gravity settler (no dispersion is produced at this step). Then, a set of four electrovalves mounted on the circuit (see Figure 1), allow to bypass the gravity settler and direct both oil and water phases in a single circuit (the water loop) at the desired concentration.

With this system, both phases flow in the aqueous phase loop and a fine dispersion of oil in water is continuously maintained by the centrifugal pump. At the end of a series of experiments, the dispersed phase fraction is measured by sedimentation of a few millilitres sample.

Flow temperature is adjusted thanks to a heat exchanger fed by a secondary heated water circuit. The temperature of all experiments has been set to $29^{\circ} \mathrm{C}$ with an accuracy of $0.1^{\circ} \mathrm{C}$. 


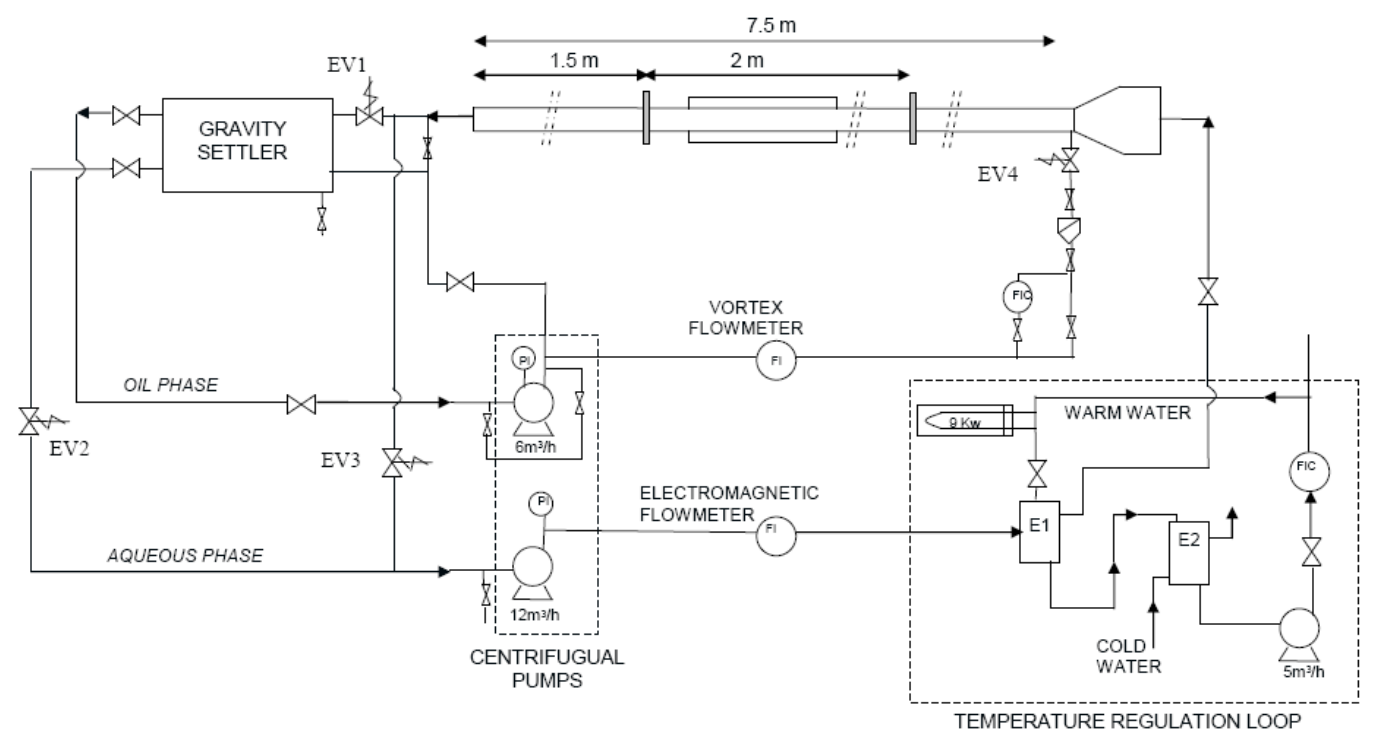

Figure 1: Schematic diagram of the two-liquid phase flow loop

\section{Instrumentation/pressure and flow measurement systems}

Pressure drop measurements were made with a differential pressure gauge. Five pressure taps were placed at $2 \mathrm{~m}$ intervals along the pipe. The instantaneous pressure signal was acquired at a frequency of $2 \mathrm{~Hz}$ and time-averaged over 1 minute time interval for each studied flow rate. At $2 \mathrm{~m}$ from the pipe inlet section, the longitudinal gradient is stable along the pipe length for both single and two-phase flow. The uncertainty on pressure drop measurement is estimated at $\pm 11 \%$.

A high speed PIV technique has been implemented to determine the 2-dimensional velocity field of the aqueous phase. A vertical laser sheet illuminates a vertical median plane of the pipe. The principle of this technique consists in the measurement of the displacement of seeding particles between two successive images of the laser sheet separated by a constant time interval. The most probable displacement of the particles between two consecutive images is calculated from the detection of the maximum of the cross-correlation function of grey level between the two images.

Fluorescent hydrophilic microparticles were used to seed the flow. These are made of poly-methyl-methacrylate (PMMA) with encapsulated Rhodamine B ( $\lambda=584 \mathrm{~nm})$. Their diameter ranges between 1 and $20 \mu \mathrm{m}$ and their density is $1.18 \mathrm{~g} / \mathrm{cm}^{3}$. Their Stokes number based on the terminal velocity is less than $10^{-4}$. Thus, they can be considered as flow tracers over the range of flow velocity investigated. The PIV system comprises a high frequency laser $(10 \mathrm{kHz})$ and a high speed camera, RS 3000 (3000frame/s at $1024 \times 1024 \mathrm{pixels}^{2}$ image resolution), equipped with a $100 \mathrm{~mm}$ focal lens. The laser source is a dual-head, diode-pumped $\mathrm{Nd}$ :YLF system $(\lambda=527 \mathrm{~nm})$. A thin laser sheet, with a thickness less than $600 \mu \mathrm{m}$, is generated 
by a divergent lens mounted on the laser head. A $45^{\circ}$ degree mirror directs the laser sheet in the vertical median plane of the pipe. The use of a high-pass filter mounted on the camera objective enables to collect only the light issued from the fluorescent micro-particles $(\lambda=584 \mathrm{~nm})$. Laser and camera are synchronized and controlled by a Processor Time Unit (PTU). Recorded images are divided into square windows within which the grey level crosscorrelation function between two consecutive images is calculated along vertical and transverse directions (using DaVis 7 software). The vector field is calculated according to a 3step iteration process with decreasing window size. The initial interrogation window is $64 \times 64$ pixels $^{2}$ and the final is $32 \times 32$ pixels $^{2}$ with a $50 \%$ overlap. This method allows a good spatial resolution of the vector field. The time between two successive images has been chosen in order to have a displacement of about one-fourth of the final window size. Statistical averages were performed over 2000 images corresponding to an integration time of about $4 \mathrm{~s}$ (acquisition frequency of $500 \mathrm{~Hz}$, double pulse). Gaussian sub-pixel interpolation is applied to optimize the computation of the displacement, a necessary refinement for accurate measurement of low velocities near the pipe wall. ${ }^{23}$ Image size is $1024 \times 1024$ pixels ${ }^{2}$, corresponding to a field size of about $50 \times 50 \mathrm{~mm}^{2}$. The enlargement factor is estimated with a graduated transparent rule placed inside the duct. PIV measurements were realized $3.3 \mathrm{~m}$ after the duct entry.

\section{Drop size}

The drop size distribution was analyzed with a laser granulometer (Mastersizer 2000). A sample is directly taken from the pipe and diluted in water to increase the refractive index difference between dispersed and continuous phase. Figure 2 shows the drop size distribution for $\phi=0.08$ and 0.12 . The drop size distribution is approximately the same for both concentrations. For $0.05<\phi<0.25$, the mean Sauter drop diameter $\left(d_{32}\right)$ is about $25 \mu \mathrm{m}$. When $\phi>0.25$, the drop size distribution could not be measured because of the instability of the sample at high concentration. We have assumed that the mean drop size produced by the pump for higher concentration remains of the same order. The effect of the pump rotation speed was also investigated. No significant influence on drop size distribution was observed in the range of rotation speed studied $(1800<N<2400 \mathrm{rpm})$. The mean diameter of the drops is smaller than the Kolmogorov length scale $\left(\eta=\left(v^{3} / \bar{\varepsilon}\right)^{1 / 4}\right)$ in the turbulent regime. Based on the Kolmogorov time scale, the drop Stokes number is much smaller than unity, suggesting that the effective viscosity concept is valid in such an emulsion flow. Moreover, based on the 
estimation of critical Capillary number and drainage time to interaction time ratio, no rupture or coalescence is expected in the pipe flow. The same drop distribution of a sample of the dispersed flow (at $\phi=0.08$ ) was observed at the pipe inlet and $2 \mathrm{~m}$ after the pipe inlet, validating this assumption.

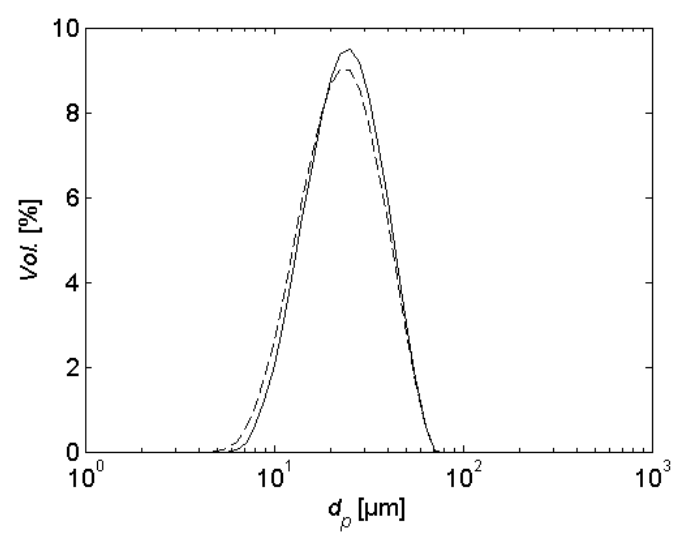

Figure 2: Drop size distribution for $\phi=0.08$ (一) and $\phi=0.12$ (--)

\section{Flow homogeneity}

A homogeneous dispersed flow is a fully dispersed flow with no mean gradient of the concentration across the pipe section. Flow homogeneity was evaluated from the radial profile of grey level in the raw images of the laser sheet. This grey level profile (averaged over 200 images) in the cross-section results from the light intensity diffused by the fluorescent microparticles. Figure 3a shows the PIV raw image of a single phase flow. On this image, white spots represent the location of the micro-particles. The corresponding grey level profile is shown in Figure 3b. The vertical (radial) intensity profile is rather flat, indicating that fluorescent particles are homogeneously distributed in the aqueous phase over the crosssection. The small gradient of intensity is only due to the non-homogeneity of the laser sheet and reflections at the pipe bottom.

A two-phase flow PIV raw image at high velocity $\left(U_{m}=1.2 \mathrm{~m} / \mathrm{s}\right)$ appears to be identical to that of the single phase flow (Figure $4 \mathrm{a})$. Due to their small size $(25 \mu \mathrm{m}$ corresponds to less than 1 pixel), oil drops cannot be detected in the image. The grey level profile (Figure $4 \mathrm{~b}$ ) is practically as flat as the single phase flow one. The dispersion can thus be considered to be homogeneous.

At low velocity $\left(U_{m}=0.28 \mathrm{~m} / \mathrm{s}\right)$, a layer of concentrated micro-particles develops in the upper part of the wall, suggesting the occurrence of partial stratification (Figure 5a). In this case, there is an increase of grey level near the top wall (Figure 5b). This is expected to be due 
to the fact that, when settling in the upper part of the pipe and forming a dense layer, oil drops capture micro-particles like in a flotation process. As such a layer is free of turbulence as shown by Conan et al. ${ }^{22}$ and the mean velocity rapidly cancels, the concentration remains stable in that layer and higher than in the bulk. Images at others velocities (not shown) indicated that the thickness of the dense layer decreases as the flow velocity increases. This layer is probably composed of the largest drops of the size distribution.
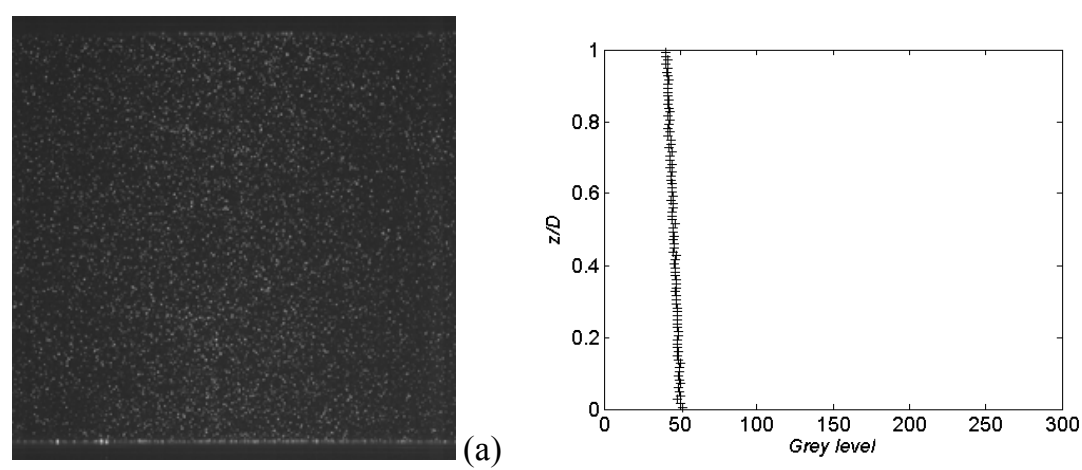

(b)

Figure 3: Single phase flow (a) PIV raw image (b) vertical grey level profile $\left(U_{m}=1.2 \mathrm{~m} / \mathrm{s}\right)$
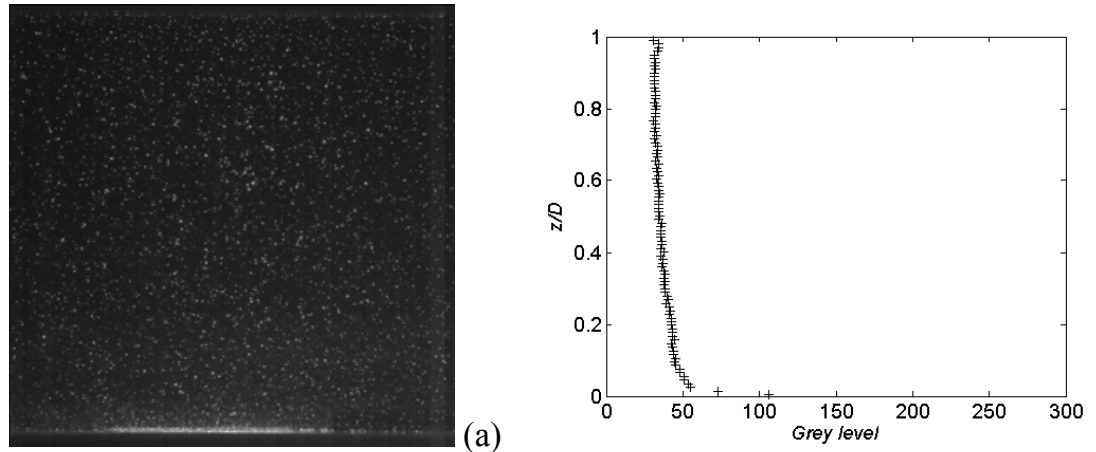

(b)

Figure 4: Homogeneous dispersed flow (a) PIV raw image (b) vertical grey level profile ( $\phi=0.21$,

$$
\left.U_{m}=1.2 \mathrm{~m} / \mathrm{s}\right)
$$
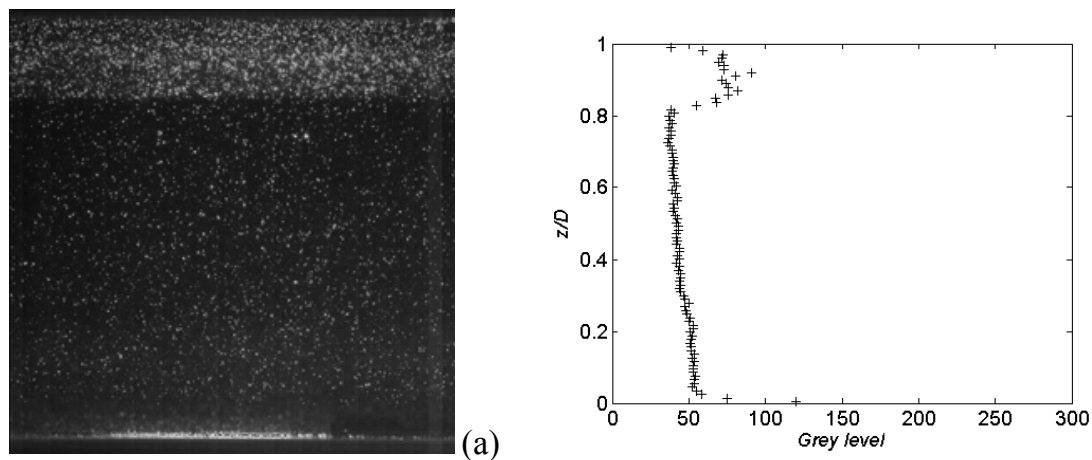

(b)

Figure 5: Partially stratified flow (a) PIV raw image (b) vertical grey level profile ( $\phi=0.21, U_{m}=0.28 \mathrm{~m} / \mathrm{s}$ ) 
Experiments were performed over a wide range of flow mixture velocity $\left(0.28<U_{m}<1.2 \mathrm{~m} / \mathrm{s}\right)$ and phase volume fraction $(0.08<\phi<0.56)$. A flow pattern map of all these experiments is given in Figure 6. This map represents the transition between homogeneous and partially stratified flow. Open symbols correspond to fully dispersed flow. Full symbols represent non homogeneous flow, when a dense layer of oil drop appears near the upper wall.

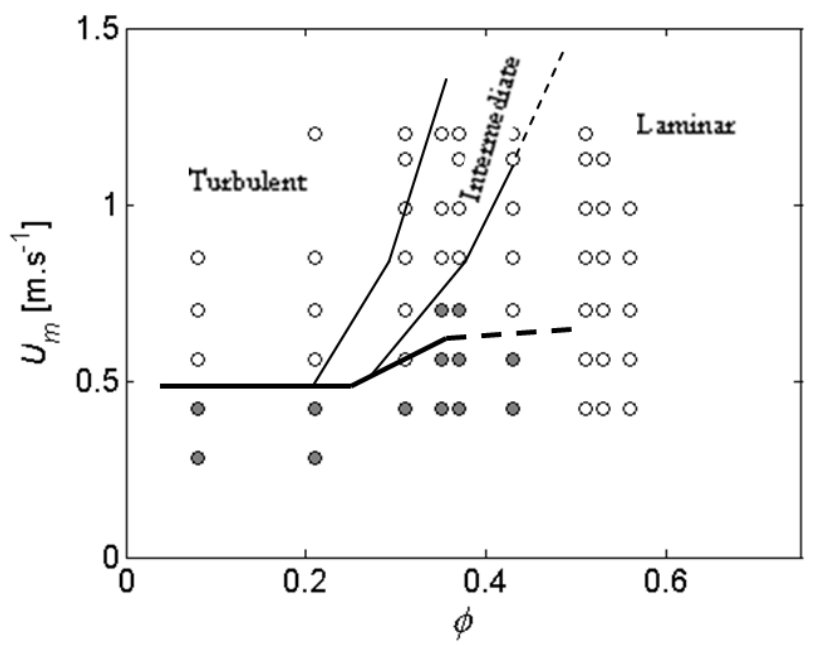

Figure 6: Flow pattern map $(\circ)$ : homogeneous flow, $(\bullet)$ : partially stratified flow. Thin lines separate the flow regime domains. Thick line is the approximate limit between the homogeneous (above) and the partially stratified configuration (below)

In Figure 6, the map of the different flow regimes investigated has been also reported, (turbulent, intermediate and laminar). These flow regimes have been identified at steady state by a combination of measurements of velocity field and pressure drop, both presented in the next section. We note that all flow regimes meet the condition of homogeneous flow configuration, each of them in a given range of mixture velocity and dispersed phase concentration.

If the condition for flow homogeneity could be expressed as a function of a critical Froude or Richardson number in turbulent (and to some extent in the intermediate) regime, the stability of such a flow configuration in laminar regime is questionable. Indeed, in this regime, there are no dynamic pressure forces that prevent the drops from settling, leading to flow stratification. Shear induced agitation is weak in the present case, especially in that range of concentration and cannot counteract the buoyancy force. ${ }^{24}$ Therefore the emulsion homogeneous flow is unstable in laminar regime and drops will segregate. However, based on the simple calculation of the settling velocity, it is possible to estimate the vertical displacement of the largest drops of the size distribution in the time interval between two successive passages of the flow in the pump (of the order of $16 \mathrm{~s}$ at $1 \mathrm{~m} / \mathrm{s}$ ). With a maximum drop diameter of $75 \mu \mathrm{m}$ (see Figure 2), this displacement is of the order $D / 10$. At high 
concentration, however, multiplying the emulsion viscosity by a factor 10 is equivalent to dividing the largest drop settling velocity and its displacement by the same factor. This is the reason why homogeneous laminar flow can be considered as stable at the scale of the present experiments. It also explains that the discrimination between homogeneous and partially stratified flow was hardly detectable in the range of concentration studied.

The results for the different flow regimes are described in the next section.

\section{Results}

\section{Turbulent regime}

The velocity field PIV measurements were first performed with single phase flow (water/glycerin mixture). For $U_{0}>0.28 \mathrm{~m} / \mathrm{s} \quad\left(R e_{0}>4800\right)$, it was verified that the flow is established and parallel at $3.3 \mathrm{~m}$ from the pipe inlet. The longitudinal mean velocity $\left(V_{x}\right)$ profile follows the expected trend in turbulent regime. An example of the radial profile of $V_{x}$ is plotted in Figure 7 (cross symbols) at a mean velocity $U_{0}=0.42 \mathrm{~m} / \mathrm{s}\left(R e_{0}=7200\right)$.

The universal power law in turbulent pipe flow reads:

$$
V_{x} / V_{x \max }=(1-r / R)^{1 / n}=\left(1-2\left|z / D-\frac{1}{2}\right|\right)^{1 / n}
$$

where $V_{x \max }$ is the velocity at the centre line and $r=|z-R|$. In equation (1), the exponent $(1 / n)$ slightly varies with the Reynolds number. ${ }^{25}$ For $R e_{0}=7400,1 / n$ is equal to $2 / 13$ (n=6.5), and the corresponding profile has been reported in Figure 7 (dashed line). The power law does well compare with the experimental data. By integrating (1) over the cross-section, we can also compare velocity at the centre line $V_{x \max }$ :

$$
V_{x \max }=U_{0}(1+1 / n)(1+1 / 2 n)=0.52 \mathrm{~m} / \mathrm{s} \text { with } n=13 / 2
$$

which gives a $3.3 \%$ relative difference with the experimental value $(0.54 \mathrm{~m} / \mathrm{s})$.

Homogeneous turbulent two-phase flows were observed for $0.56<U_{m}<1.2 \mathrm{~m} / \mathrm{s}$ and $0.08<\phi<0.21$ and for $U_{m}>0.85 \mathrm{~m} / \mathrm{s}$ and $\phi=0.31$ (see flow map of Figure 6). The normalized longitudinal velocity vertical (radial) profile has been reported in Figure 7 at two concentrations $\left(\phi=0.08, U_{m}=0.56 \mathrm{~m} / \mathrm{s}\right.$ and $\left.\phi=0.21, U_{m}=0.85 \mathrm{~m} / \mathrm{s}\right)$. The two velocity profiles are quite symmetrical (confirming flow homogeneity) and almost identical to the single phase flow case. Applying Eq. 2 to these profiles gives $V_{x \max }=0.70 \mathrm{~m} / \mathrm{s}$ and $1.06 \mathrm{~m} / \mathrm{s}$, which are close to the experimental values (with a relative difference of $2.7 \%$ and $3.6 \%$ respectively). 


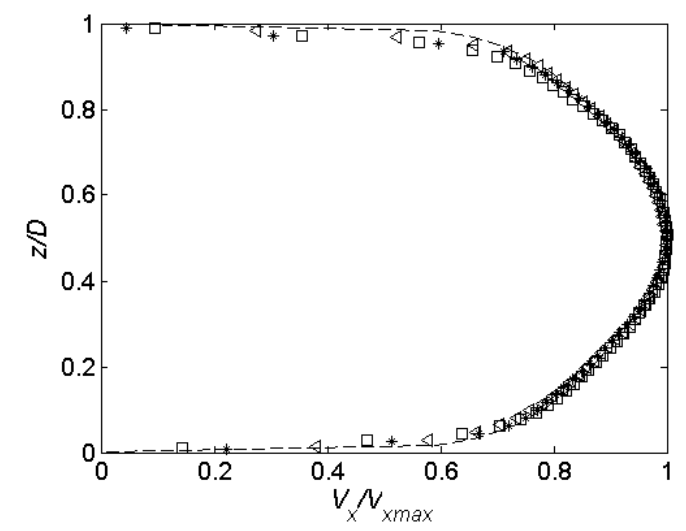

Figure 7: Normalized longitudinal velocity profile. Single phase flow $(*):\left(U_{0}=0.42 \mathrm{~m} / \mathrm{s}\right.$, $\left.R e_{0}=7200, V_{x m a x}=0.54 \mathrm{~m} / \mathrm{s}\right)$; Two-phase flow $(\triangleleft):\left(\phi=0.08, U_{m}=0.56 \mathrm{~m} / \mathrm{s}, V_{x \max }=0.72 \mathrm{~m} / \mathrm{s}\right),(\square)$ : $\left(\phi=0.21, U_{m}=0.85 \mathrm{~m} / \mathbf{s}, V_{x \max }=1.1 \mathrm{~m} / \mathbf{s}\right) ;(--): V_{x} / V_{x \max }=(1-2 \mid z / D-1 / 2)^{2 / 13}$

For these three cases (single phase and the two dispersed flows), the wall law is displayed in Figure 8a, scaling the axial mean velocity by the wall friction velocity $v^{*}$, and the distance from the wall $(\delta)$ by the ratio $v^{*} \rho_{c} / \mu_{c}$, where $\mu_{c}$ and $\rho_{c}$ are the continuous phase dynamic viscosity and density (i.e. at $\phi=0$ ). The friction velocity is deduced from pressure drop measurements in each of these flows:

$$
v^{*}=\sqrt{\frac{R}{2} \frac{1}{\rho_{m}}\left|\frac{d P}{d x}\right|}
$$

where $\rho_{m}=\phi \rho_{d}+(1-\phi) \rho_{c}$ is the mixture density of two-phase flows. In single-phase turbulent pipe flow, the velocity profile near the wall is given by the universal log-law in the inertial layer:

$$
\delta^{+} \geq 30 \quad V^{+}=\frac{1}{\kappa} \ln \left(\delta^{+}\right)+5.5
$$

where $\kappa$ is equal to 0.41 , and the linear law is valid in the viscous layer

$$
0 \leq \delta^{+} \leq 5 \quad V^{+}=\delta^{+}
$$

with $\delta^{+}=\delta v^{*} \rho_{c} / \mu_{c}$.

The curves of Figure 8a clearly indicate that the single phase flow (crosses) follows the theoretical profiles in both layers up to $\delta^{+}=100$ (for larger values of $\delta^{+}$, the experimental velocity data are slightly above the log-law). In the same interval $\left(0 \leq \delta^{+} \leq 100\right)$, two-phase velocity profiles are shifted to the left, and the shift increases with the dispersed phase concentration. Such behaviour suggests an influence of the effective viscosity of the mixture. 

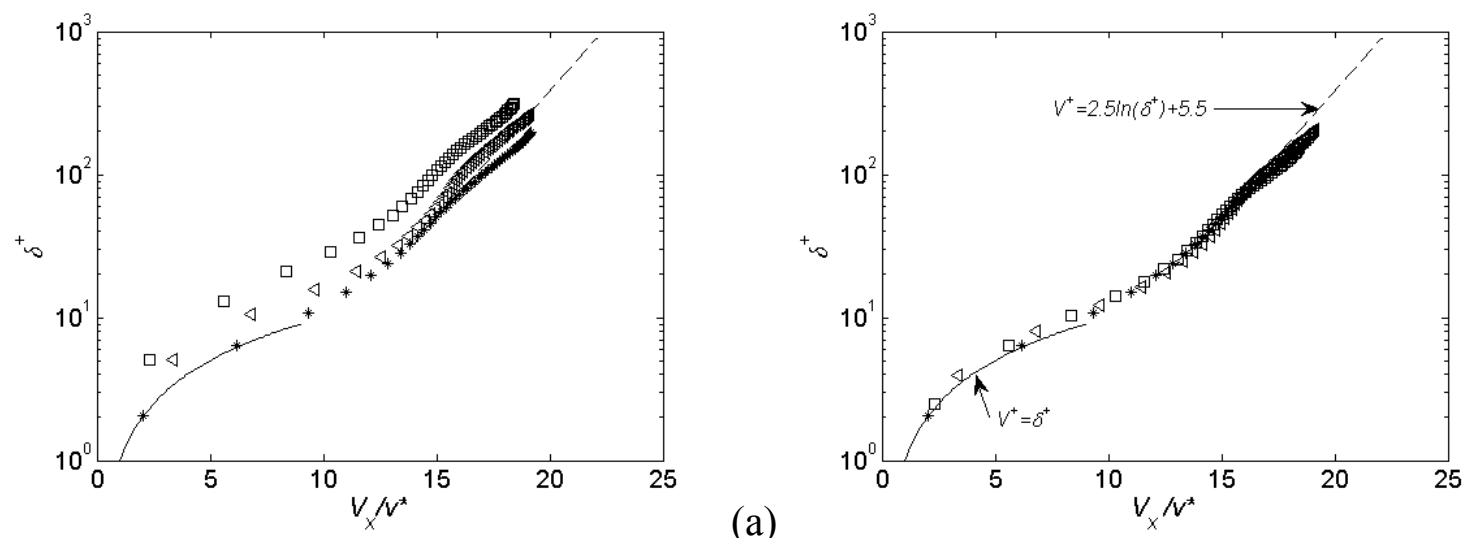

(a)

Figure 8: Wall law velocity. Single phase flow (*⿻丷木): $\left(U_{0}=0.42 \mathrm{~m} / \mathrm{s}, R e_{0}=7200, V_{x \max }=0.54 \mathrm{~m} / \mathrm{s}\right)$;

Two-phase flow $(\triangleleft):\left(\phi=0.08, U_{m}=0.56 \mathrm{~m} / \mathrm{s}, V_{x \max }=0.72 \mathrm{~m} / \mathrm{s}\right),(\square):\left(\phi=0.21, U_{m}=0.85 \mathrm{~m} / \mathrm{s}\right.$, $V_{x \max }=1.1 \mathrm{~m} / \mathrm{s}$ ). (a) $\delta$ normalized by continuous phase properties, $\mu_{c}$ and $\rho_{c}$ (b) $\delta$ normalized by mixture properties, $\mu_{m}$ and $\rho_{m}$

Indeed, it is possible to match the two-phase velocity profiles with that of the singlephase, through a simple multiplication of each two-phase profile by a given factor. Doing so, two-phase flow data collapse on a single curve, as illustrated in Figure 8b. This multiplication amounts to replacing the continuous phase viscosity $\mu_{c}$ in Eqs. 4 and 5 by a mixture viscosity $\mu_{m}$, equal to $4.10^{-3} \mathrm{~Pa}$.s with $\rho_{m}=1068 \mathrm{~kg} / \mathrm{m}^{3}$ for $\phi=0.08$ and $\mu_{m}=610^{-3} \mathrm{~Pa}$.s with $\rho_{m}=1014 \mathrm{~kg} / \mathrm{m}^{3}$ for $\phi=0.21$. These results show that in turbulent regime, the liquid-liquid emulsion behaves as a single fluid with mixture properties. They are also consistent with the results of Faruqui and Knudsen ${ }^{19}$ who first used this method. According to these results, the cross-section integrated momentum balance can be applied to the mixture:

$$
-\frac{d P}{d x}=f_{m} \frac{\rho_{m} U_{m}^{2}}{R}
$$

where $f_{m}$ is the mixture wall friction factor. For fully turbulent flow in smooth pipe, $f_{m}$ is given by Blasius' law (in the range of Reynolds number studied $\left(<1210^{4}\right)$, pipe roughness has no influence on wall friction factor: ${ }^{26}$

$$
f_{m}=0.079 R e_{m}^{-1 / 4}
$$

The mixture Reynolds number is given by:

$$
R e_{m}=\rho_{m} U_{m} D / \mu_{m}
$$

where $\mu_{m}$ is the effective dynamic viscosity of the dispersion, a priori unknown. Therefore, the evolution of the pressure drop as a function of mean velocity reads:

$$
-\frac{d P}{d x}=A \times U_{m}^{1.75} \text { with } A=\left(\frac{0.067 \rho_{m}^{0.75} \mu_{m}^{0.25}}{R^{1.25}}\right)
$$


According to Eq. 9, the pressure gradient is proportional to the mixture velocity to the power 1.75 and the proportionality coefficient $A$ varies as $\mu_{m}^{1 / 4}$. In Figure $9 \mathrm{a}$ and $9 \mathrm{~b}$, the evolution of the pressure drop as a function of the mixture velocity is reported at two concentrations, 0.08 and 0.21 in the turbulent regime. Both curves can be well fitted by a 1.75 power law, suggesting that Blasius' equation is valid.
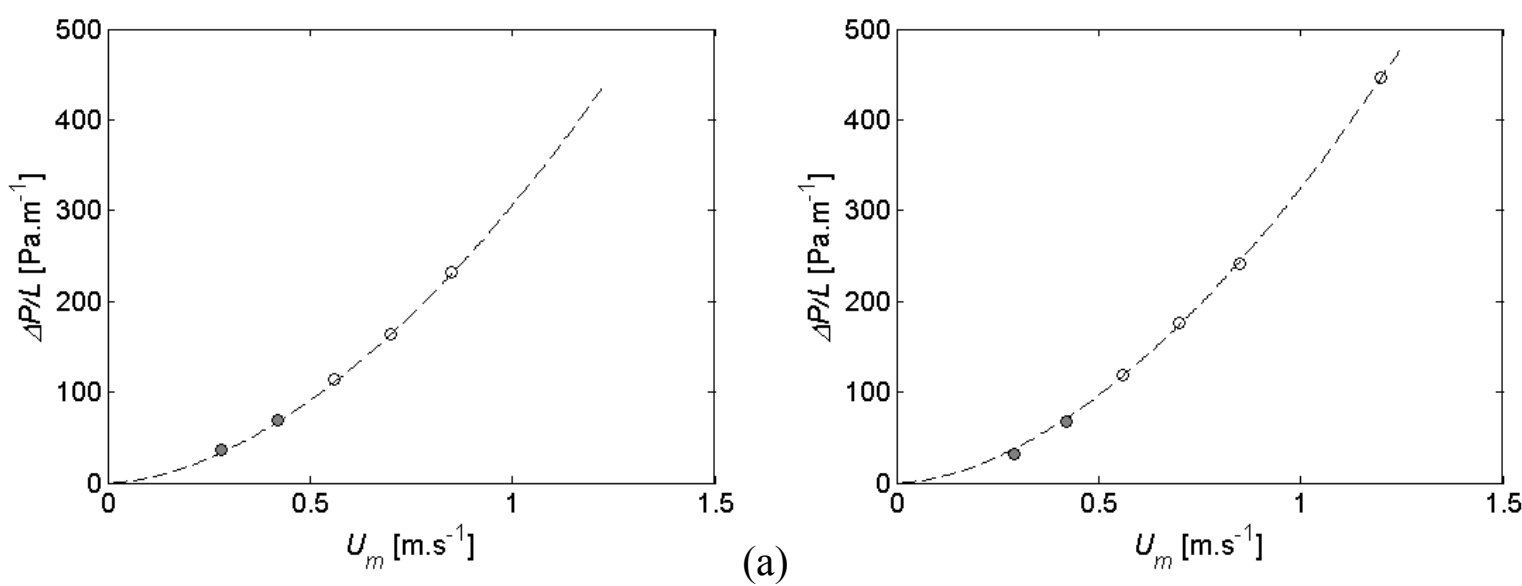

Figure 9: Evolution of the pressure drop as a function of the mixture velocity for (a) $\phi=0.08$, (b) $\phi=0.21$. Solid symbols correspond to partially homogeneous profiles. Dashed line corresponds to Eq. 9.

Note that on these graphs, full symbols correspond to partially stratified regime at low velocity and do not deviate much from the pressure drop law. This is due to the weak value of the stratified layer thickness $\delta_{s}$. It can be shown that the main contribution to the correction of the pressure gradient is of the order of $\left(1+\frac{1}{2 \pi}\left(2 \delta_{s} / R\right)^{3 / 2}\right)$, which represents only a few percent in the present case, well within the range of measurement uncertainty.

For each concentration, the dynamic viscosity of the emulsion can be deduced from Eq. 9. Corresponding values are reported in Table 2. Despite the great sensitivity of the mixture viscosity to the value of $A$ in Eq. 9 (and consequently to the pressure drop measurement uncertainty), the identified values of the effective viscosity are close to the former values derived from the matching of the log law. The values predicted by Vand's equation $^{27}$ for diluted suspensions $\left(\mu_{m}=\mu_{c}\left(1+2.5 \phi+7.35 \phi^{2}\right)\right.$ ), are also reported in this table. They correctly fit the values deduced from the log-law. Note that the models of Manley and Mason, ${ }^{28}$ Krieger and Dougherty ${ }^{29}$ or Batchelor ${ }^{30}$ predict similar values (comparison made but not shown). It can be concluded that in turbulent regime and in this range of concentration, the dispersion behaves as a suspension of non-inertial hard spheres. 
Table 2: Effective viscosity values deduced from velocity log-law, Blasius' law and Vand's equation

$$
\mu_{m} \text { (Pa.s) }\left\{\begin{array}{lcc}
\text { Concentration } \phi & 0.08 & 0.21 \\
\text { (Velocity) log-law } & 4.10^{-3} & 6.10^{-3} \\
\text { (Pressure drop) Blasius' law } & 3.710^{-3} & 5.510^{-3} \\
\text { Vand (1948) } & 4.10^{-3} & 5.910^{-3}
\end{array}\right.
$$

Such behaviour is consistent with the evaluation of the deformation of the drops in the turbulent flow. Based on the single-phase flow properties and the average turbulent energy dissipation rate $\left(\bar{\varepsilon} \cong 5.610^{-3}\left(U_{0}^{3} / D\right)\right.$ ), the Kolmogorov length scale $\left(\eta=\left(v_{c}^{3} / \bar{\varepsilon}\right)^{1 / 4}\right)$ is about $120 \mu \mathrm{m}$ at $U_{0}=1 \mathrm{~m} / \mathrm{s}$, and is significantly larger than the drop diameter $(\sim 25 \mu \mathrm{m})$. The deformation seen by the drops is therefore induced by the viscous shear rate $\dot{\gamma}_{k}=\left(2 \bar{\varepsilon} / 15 v_{c}\right)^{1 / 2}$. Based on that scale, the capillary number $C a=\mu_{c} \dot{\gamma}_{k} \bar{r}_{32} / \sigma$ is found to be the order of $10^{-4}$, leading to the conclusion that the drops are not deformed.

\section{Laminar regime}

At high dispersed phase fraction $\phi \geq 0.51$ and $0.56<U_{m}<1.2 \mathrm{~m} / \mathrm{s}$, the flow becomes laminar (see Figure 6). Two vertical profiles of the longitudinal velocity are illustrated in Figure 10 at a concentration of $\phi=0.53$, and two mixture velocities, $U_{m}=0.56$ and $0.7 \mathrm{~m} / \mathrm{s}$. These profiles are quite symmetrical. They are well fitted by the normalized parabolic profile in a tube:

$$
V_{x} / V_{x \max }=1-(r / R)^{2}=1-4\left(z / D-\frac{1}{2}\right)^{2} \text { with } V_{x \max }=2 \times U_{m}
$$

The relative accuracy on the maximum velocity is $1.7 \%$ and $7 \%$ at $U_{m}=0.56$ and $0.7 \mathrm{~m} / \mathrm{s}$ respectively. This result suggests that the concentrated emulsion behaves as a Newtonian fluid with an effective viscosity that can be determined using the pressure drop-mean velocity curve displayed in Figure 11. On this figure, the pressure gradient varies linearly with the mixture velocity. In this flow regime, the friction factor is therefore given by HagenPoiseuille law:

$$
f_{m}=16 / R e_{m} \text { with } R e_{m}=\rho_{m} U_{m} D / \mu_{m}
$$

And the slope is directly proportional to the effective viscosity:

$$
\mu_{m}=\left|\frac{d P}{d x}\right| \frac{R^{2}}{8 U_{m}}
$$


The viscosity value deduced from Eq. 12 can be compared with those obtained from the velocity measured at the centre line $V_{x \max }$ (the relative difference will be the same as that between $V_{x \max }$ and $2 \times U_{m}$ which is very low). For three different concentrations in the laminar regime, these values are reported in Table 3. At this concentration, the effective viscosity is an order of magnitude higher than the continuous phase viscosity $\left(3.210^{-3} \mathrm{~Pa} . \mathrm{s}\right)$ and is a rapidly growing function of the concentration. Values predicted by the Eilers correlation for liquidliquid emulsions (Eq. 17) are also reported. The correlation does fit our data very well, the discrepancy being well below the pressure drop measurement uncertainty.

Table 3: Effective viscosity in laminar regime at high concentration

$\mu_{m}$ (Pa.s) $\left\{\begin{array}{llll}\text { Concentration } \phi & 0.51 & 0.53 & 0.56 \\ \text { (Eq.12) } & 0.03 & 0.033 & 0.045 \\ \left.\text { (Eq.12, } V_{x \max }\right) & 0.031 & 0.035 & 0.049 \\ \text { Eilers (1941) } & 0.03 & 0.036 & 0.048\end{array}\right.$

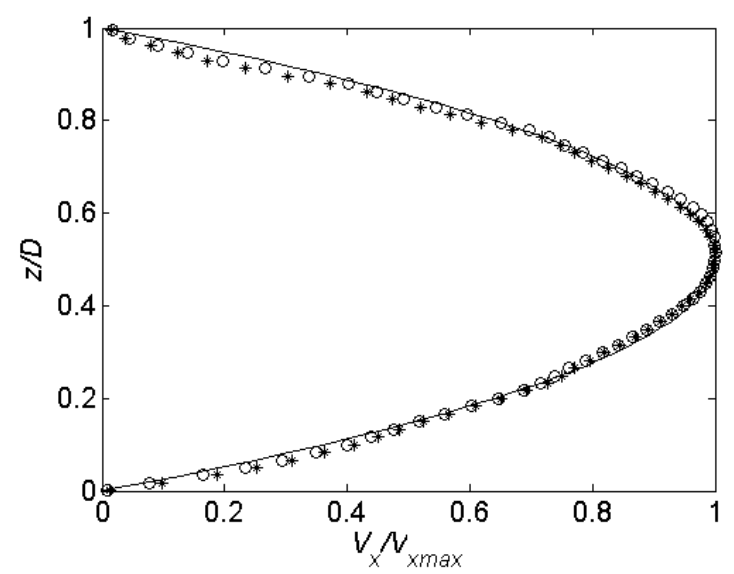

Figure 10: Normalized velocity profile at $\phi=0.53,(*) U_{m}=0.56 \mathrm{~m} / \mathrm{s}\left(V_{x m a x}=1.1 \mathrm{~m} / \mathrm{s}\right),(\circ) U_{m}=0.7 \mathrm{~m} / \mathrm{s}$ $\left(V_{x \max }=1.3 \mathrm{~m} / \mathrm{s}\right),(-)$ parabolic profile

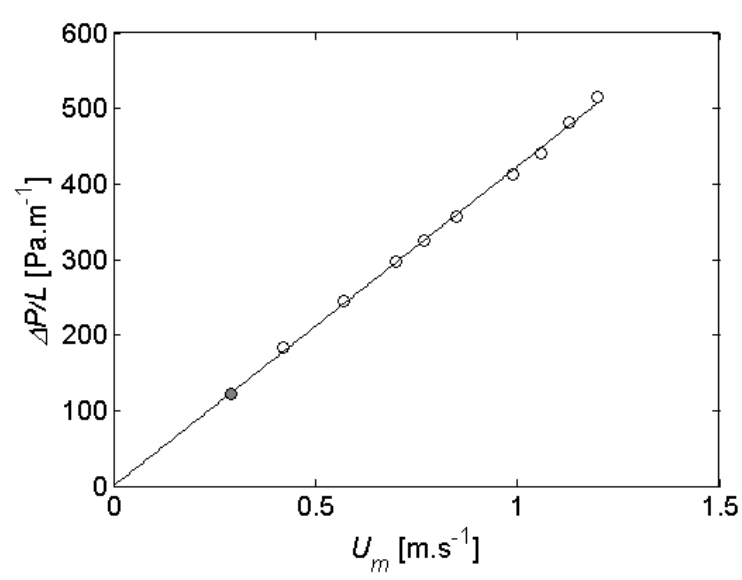

Figure 11: Evolution of the pressure drop as a function of the mixture velocity at $\phi=0.53$ 
As for the turbulent regime, it seems relevant to verify that the Newtonian behaviour of these dispersions is consistent with a spherical shape of the drop. In laminar flow, the maximum capillary number (based upon the mixture viscosity) can be calculated from the velocity gradient at the wall:

$$
C a_{\max }=\mu_{m} \dot{\gamma}_{\max } \bar{r}_{32} / \sigma \text { with } \dot{\gamma}_{\max }=4 U_{m} / R
$$

For a mean velocity of $1.1 \mathrm{~m} / \mathrm{s}$ and an effective viscosity of $0.05 \mathrm{~Pa} . \mathrm{s}$, the maximum value of the capillary number is $3.610^{-3}$, suggesting that the drops remain spherical.

Note that the laminar Newtonian regime has been verified for a concentration as high as 0.56 . Measurements were performed at a concentration of $70 \%$ and the velocity profiles clearly exhibited a non-Newtonian behaviour (shear-thinning). It is interesting to point out that Conan et al. ${ }^{22}$ observed a similar behaviour of millimetre-size dispersions (with the same phase system) for concentrations larger than 0.6. Hence, it seems reasonable to admit that, in the absence of other compounds and strong interfacial forces (the concentration of seeding micro-particles is too small to have any effect on the dispersion rheology), the Newtonian behaviour of non-Brownian homogeneous liquid-liquid dispersions is expected to be valid for concentrations as high as $0.55-0.6$, provided that the capillary number is small. This condition will not be fulfilled in a laminar pipe flow if the Capillary number is greater than 0.1 (which corresponds to a small finite deformation of the drop) and the Reynolds number below the critical Reynolds number ( $\operatorname{Re}_{\text {crit }} 2100$ in single phase flow), leading to a critical value of an Ohnesorge number defined as:

$$
O h=\frac{\mu_{c}}{\sqrt{\rho_{c} \sigma \overline{r_{32}}}}>O h_{c r i t}=0.31 \frac{D}{r_{32}} \frac{g(\phi)}{\sqrt{\operatorname{Re}_{\text {mcrit }}}} \text { with } g(\phi)=\frac{\rho_{c}}{\rho_{m}} \frac{\mu_{c}}{\mu_{m}}
$$

In this relation, the function $g(\phi)$ is given by the Newtonian behaviour of the emulsion. For values of the Ohnesorge number larger than this critical value, the drops will be deformed and the emulsion behaviour will no longer be Newtonian. This will be the case in particular with small diameter tubes, high concentration $(<0.65)$ and high continuous phase viscosity. Note that this range is limited by the occurrence of shear induced drop fragmentation, occurring at critical capillary numbers of the order of unity, for phase viscosity ratios between $10^{-2}$ and $10 .^{31} \mathrm{~A}$ similar calculation can be made in turbulent regime for drops size smaller than the Kolomogorov length scale, using a critical value of the Capillary number based upon the viscous range shear stress and the emulsion viscosity. Using classical scaling law of fully developed turbulent pipe flow, the condition for having deformed drops in turbulent flow without breaking will be given by: 


$$
0.1 \frac{D}{r_{32}}<0.0274 \sqrt{R e_{m}} C a_{m}<\frac{D}{r_{32}} \text { with } C a_{m}=\frac{\mu_{m} U_{m}}{\sigma} \text { and } R e_{m}=\frac{\rho_{m} U_{m} D}{\mu_{m}}
$$

Small pipe diameter and high velocity will also favour drop deformation in that regime. As expected, the effect of the mixture viscosity is weaker than in laminar regime.

\section{Intermediate regime}

In a narrow range of mean velocity and concentration, the flow regime is neither laminar nor turbulent. This regime occurs for $0.35<\phi<0.45,0.56<U_{m}<1.2 \mathrm{~m} / \mathrm{s}$ and for $\phi=0.31, U_{m}<0.85 \mathrm{~m} / \mathrm{s}$ (see Figure 6). Mean velocity profiles have different shapes depending on mixture velocity and phase fraction. Two typical mean velocity profiles measured in this regime are reported in Figure 12a and 12b for two different concentrations at the same mean velocity $\left(\phi=0.43, U_{m}=1.2 \mathrm{~m} / \mathrm{s}\right)$ and $\left(\phi=0.37, U_{m}=1.2 \mathrm{~m} / \mathrm{s}\right)$. The mean velocity profiles of Figure 12 are clearly not symmetrical. This shape is due to the fact that the average values do not converge over a $4 \mathrm{~s}$ integration time scale, and a much longer integration time length should be chosen in that case. This phenomenon is attributed to the alternation of laminar and turbulent flow sections at low frequency, characteristic of this intermediate regime. ${ }^{25} \mathrm{We}$ can observe on these graphs that the velocity profile of Figure 12a is closer to a parabolic profile while that of Figure $12 \mathrm{~b}$ is closer to a turbulent profile, the maximal velocity being smaller in the latter case. Considering the increase of mixture viscosity with the dispersed phase concentration (due to the effective viscosity), the Reynolds number would be lower for $\phi=0.45$ than for 0.37 which is coherent with the velocity profiles shapes. These results confirm the validity of the effective viscosity concept in the intermediate regime.
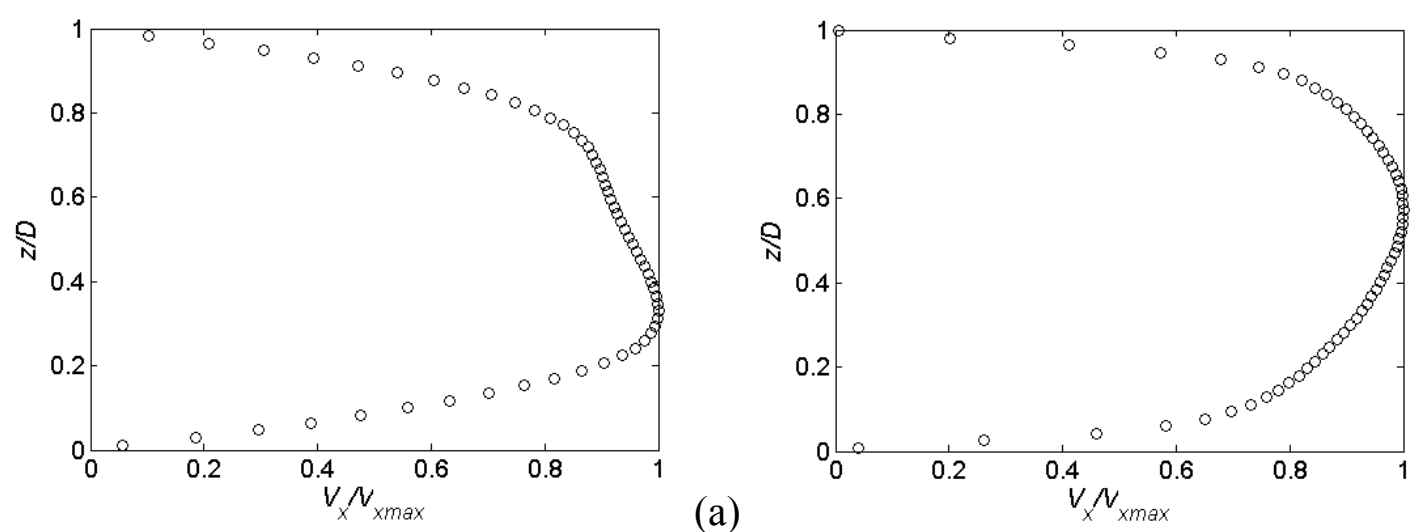

Figure 12: Axial velocity profile (a) $\phi=0.43, U_{m}=1.2 \mathrm{~m} / \mathrm{s}, V_{x \max }=1.8 \mathrm{~m} / \mathrm{s}$ (b) $\phi=0.37, U_{m}=1.2 \mathrm{~m} / \mathrm{s}$, $V_{x \max }=1.6 \mathrm{~m} / \mathrm{s}$ 


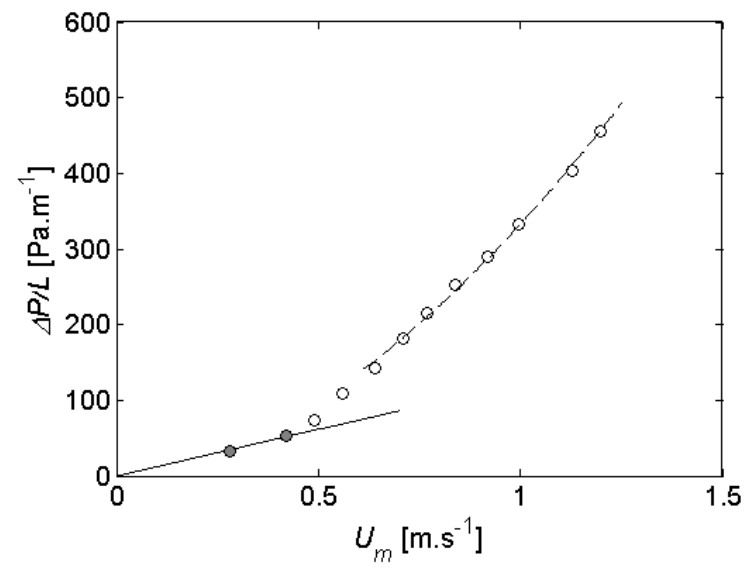

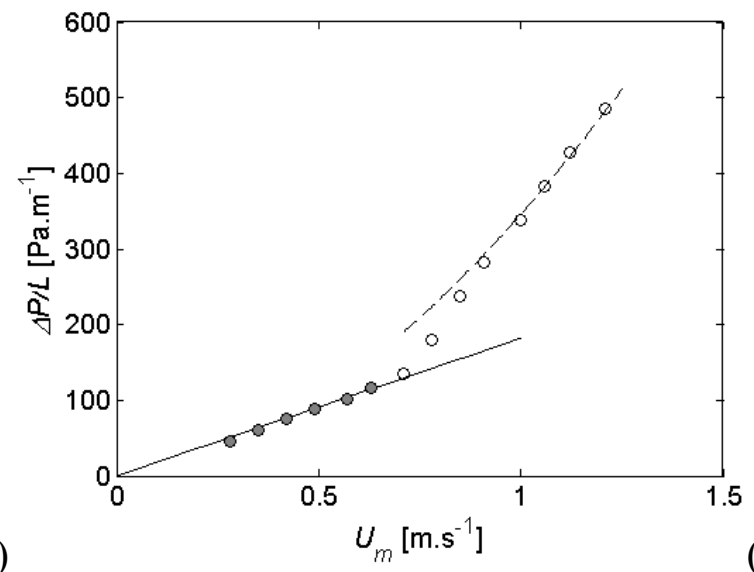

(b)

Figure 13: Pressure drop as a function of $\boldsymbol{U}_{\boldsymbol{m}}$ (a) $\phi=0.31$ (b) $\phi=0.36,(-) d P / d x \propto U_{m}$ (--) $d P / d x \propto U^{1.75}$

For intermediate flow regime, effective viscosities were deduced only from pressure drop measurements. The evolution of the pressure drop in the intermediate regime is characterized by a transition between a linear behavior at low velocity to a power law behavior at higher velocities, as illustrated in Figures 13a and 13b. There is no explicit wall friction law for intermediate regime but the mixture viscosity can be determined with either the HagenPoiseuille (Eq. 11) or the Blasius (Eq. 9) law, depending on mixture velocity. For $\phi=0.36$ (Figure 13b), pressure drop measurements are proportional to mixture velocity when $U_{m}<0.7 \mathrm{~m} / \mathrm{s}$ and fit the power law $d P / d x \propto U_{m}^{1.75}$ when $U_{m}>0.7 \mathrm{~m} / \mathrm{s}$. For $\phi=0.31$, only two points correspond to the linear (but also partially stratified) regime, whereas at $\phi=0.43$ (not shown in this paper), the linear regime is dominant. Viscosity values identified in that way are presented in the following section.

\section{Mixture viscosity model}

There are many effective viscosity models for low inertia suspensions and emulsions. ${ }^{1,16}$ In the case of Newtonian emulsions, the model of Krieger and Dougherty ${ }^{29}$ for non-Brownian monodispersed spherical particles is a reference law:

$$
\mu_{r}=\mu_{m} / \mu_{c}=\left(1-\phi / \phi_{m}\right)^{-2.5 \phi_{m}}
$$

where $\phi_{m}$ is the concentration at maximum packing, which is equal to 0.64 for a random packing. For polydispersed solid suspensions, $\phi_{m}$ may take larger values $(0.7-0.74)$. In the case of a Newtonian emulsion with non-deformed drops, the internal viscosity may be accounted for $\mathrm{Pal}^{32}$, but in most of the cases, even in the absence of surfactants, drops interfaces are immobile due to the presence of contaminants and will behave (if undeformed) 
as particles. ${ }^{33}$ As a consequence, for this type of emulsion, the viscosity ratio is not expected to play a major role. The empirical model of Eilers ${ }^{34}$ established in the case of bitumen emulsions illustrates this characteristic of a Newtonian emulsion:

$$
\mu_{r}=\frac{\mu_{m}}{\mu_{c}}=\left(1+\frac{2.5 \phi}{2\left(1-\phi / \phi_{m}\right)}\right)^{2}
$$

where $\phi_{m}$ is the maximum packing fraction of drops without deformation. In fact, the Krieger and Dougherty law and Eilers' law are very similar, they predict the mixture viscosity of Newtonian emulsion like a hard sphere suspension, with the maximum packing fraction $\phi_{m}$ being a fitting parameter accounting for polydispersity. Note also that the $\phi_{m}$ value in Eqs. 16 or 17 is purely theoretical, in the sense that, for polydispersed systems (which is always the case for liquid-liquid dispersion), the Newtonian behaviour will hold for concentrations ranging up to $0.55-0.65$. In the intermediate region of concentrated emulsions $(0.55$ $0.65<\phi<\phi_{m}$ ), Newtonian behavior is no longer valid and the apparent viscosity of the emulsion will be strongly dependent upon drop mean size and mainly upon size distribution. In that range of concentration, polydispersity will tend to reduce the emulsion viscosity and a strong shear thinning behaviour is observed. ${ }^{35}$ For concentrations larger than $\phi_{m}$ (so-called highly concentrated emulsions), drop deformation will drive the emulsion rheology, whereas below the intermediate concentration range $(\phi<0.55-0.65)$, polydispersity and drop size have no effect on the effective viscosity.

Mixture viscosity values identified in the different flow regimes are plotted as a function of the concentration in Figure 14. In this graph, full symbols and crosses represent the viscosity values determined in the turbulent and laminar regimes respectively.

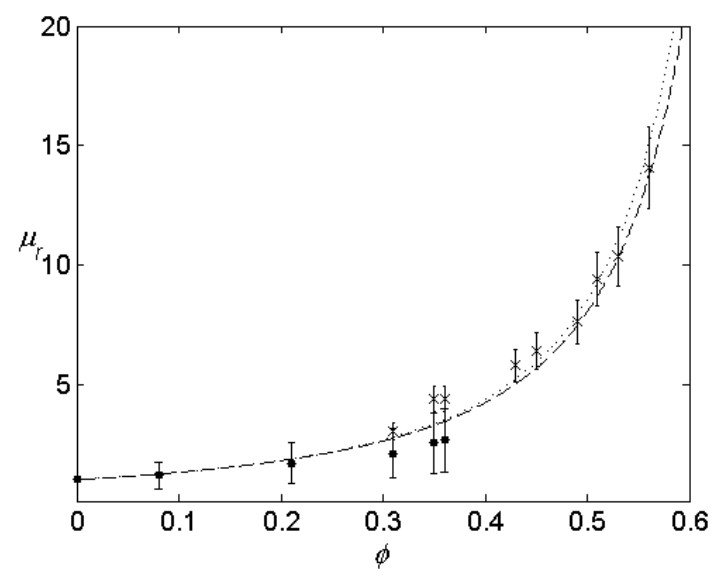

Figure 14: Relative viscosity as a function of oil volume fraction $\left(\phi_{m}=0.74\right),(\ldots)$ Eilers, (- - -) Krieger and Dougherty. $(\bullet)$ Blasius friction factor $(\times)$ Hagen-Poiseuille friction factor 
They are well fitted by Krieger and Dougherty and Eilers' laws with $\phi_{m}=0.74$, which are nearly identical. Vertical bars represent here the maximum uncertainty related to the pressure drop and flowrate measurement ( $\delta d P \approx \pm 8 \%, \delta Q= \pm 2 \%$ ), the relative weight effect of which is maximum in the turbulent regime. For intermediate flow regime, at $\phi=0.31,0.35$ and 0.36 , the apparent viscosity was determined both with Hagen-Poiseuille and Blasius' laws leading to a correct framing of the viscosity models. For $\phi=0.43$ and 0.45 when the flow tends to be laminar, the experimental values of the mixture viscosity calculated from the Hagen-Poiseuille relation are slightly underestimated by the viscosity models.

Overall, it can be concluded that Eqs. 16 and 17 give a good prediction of the effective viscosity of the flowing emulsion in all regimes, confirming the Newtonian hard sphere type behaviour of the emulsion.

\section{Wall friction factor}

Experimental values of wall friction calculated from Eq. 6 as a function of mixture Reynolds number using the viscosity model of Krieger and Dougherty (Eq. 16), are displayed in Figure 15a, b, and compared to the Hagen-Poiseuille and the Blasius laws. Friction factor data have been collected in the case of fully dispersed flows for Reynolds numbers ranging between 400 and 12,000, corresponding to concentration values between 0.08 and 0.56 . These two extreme cases do follow well the single phase flow friction law, the Hagen-Poiseuille law at low $R e_{m}$ for $\phi=0.56$ and the Blasius law at high $R e_{m}$ for $\phi=0.08$. When increasing the concentration between 0.08 and 0.56 , as expected, the friction factor evolution with $R e_{m}$ is also well predicted by these two laws.

These results confirm the early work of Baron ${ }^{16}$ in the turbulent regime, of Cengel et al. ${ }^{17}$ and Faruqui and Knudsen ${ }^{19}$ in the laminar and turbulent regimes, and of $\mathrm{Pal}^{18}$ in both laminar and turbulent regimes, in the case of surfactant stabilized emulsion of the same size range as that studied in the present work.

However, Figure 15a exhibits some differences with single-phase flow behaviour which are worth to be pointed out. These differences occur in the transition region which is shown in expanded view in Figure 15b (including additional data sets). In this graph the dashed line represents the average curve of Nikuradse ${ }^{29}$ data in single phase flow. In the transition region, this curve is above our experimental data, the closest data set corresponding to it being that of $\phi=0.31$. As the concentration is increased, experimental data move 
downward away from this curve, indicating a delay to transition towards fully developed turbulence. The matching of Blasius' law is observed for a Reynolds number close to 5000 at $\phi=0.37$ and probably for a larger Reynolds number at larger concentrations, although we could not collect data in that domain, due to the insufficient pump power. Note also that the limit of Newtonian emulsion can be reached at higher $R e_{m}$, leading to a drag reduction effect induced by the drop deformation. ${ }^{18}$ But evaluation of this regime through Eq. 15 indicates that the present range of flow parameters and the size distribution are far from this condition (with the phase system investigated in this study, beginning of drop deformation would be observed at a concentration of 0.56 and a $R e_{m}$ of $10^{4}$ ).

One can notice in Figure $15 \mathrm{~b}$ that as the concentration is increased, the laminarturbulent transition is delayed from $R e_{m} \sim 2100$ up to $R e_{m} \sim 3000$ for the highest two concentrations studied in that regime $(\phi=0.43$ and 0.45$)$. Such a result has not yet been reported for the case of a Newtonian emulsion. ${ }^{18}$ However, focussing on the laminarturbulence transition of neutrally buoyant coarse suspensions, Matas et $\mathrm{al}^{21}$ have demonstrated an influence of particle size and concentration on the critical Reynolds number. Whereas large particles tend to lower the critical mixture Reynolds number (based upon the Krieger and Dougherty viscosity model), smaller particles have no effect for concentrations up to 0.25 . Above this concentration, the critical Reynolds number tends to increase (although the authors could provide only a limited set of data for that range of concentration). Although no explanation was proposed by Matas et al., such behaviour is consistent with the present results.

In addition to this effect of concentration in the transition domain and the increase of the critical Reynolds number, a slight deviation from the Hagen-Poiseuille friction law is observed for Reynolds numbers ranging between 1500 and 3000. In this region, the absolute value of Reynolds number exponent is slightly smaller than 1, and the coefficient slightly greater than 16, resulting in an upward shift of the friction factor compared to the $16 / R e_{m}$ curve. One plausible explanation for this shift is the uncertainty in the pressure drop measurement and the difference between the exact value of the viscosity and that calculated by the Krieger and Dougherty model. However, the alignment on this curve over that range of $R e_{m}$ of three sets of data at different concentrations $(0.43,0.45$ and 0.51$)$ suggests that this trend originates from a physical mechanism. Such a mechanism has been partially identified by the analysis of velocity field in this range of $R e_{m}$. When $R e_{m}<1500$, the flow is laminar and velocity fluctuations are negligible. When $1500<R e_{m}<3000$ and $\phi \geq 0.43$, the flow is still 
laminar but large amplitude low frequency fluctuations are observed and longer integration times are necessary to obtain converged parabolic profiles. These low frequency fluctuations would explain that the slight increase of the pressure drop (which is integrated over a longer time scale) in the laminar velocity signal possibly results from the intermediate regime low frequency fluctuations, but we have no explanation for the fact that they are not dissipated in this Reynolds range, or for the effect of the concentration in the intermediate regime. It can be concluded that for a Newtonian emulsion, the critical Reynolds number increases with the concentration and the width of the mixture Reynolds range of the transition regime also increases with the concentration. Such a trend should be validated via further investigations. This problem seems to offer promising test case for numerical simulations. However, only DNS method resolved at the scale of the drop could reproduce the effective viscosity effect (such as the force coupling method by Climent and Maxey ${ }^{36}$ ). As the particle size must be kept smaller than the Kolomogorov scale, such work seems to be out of the range of present numerical capabilities (see Sundaresan et al. ${ }^{15}$ for a discussion on this topic).
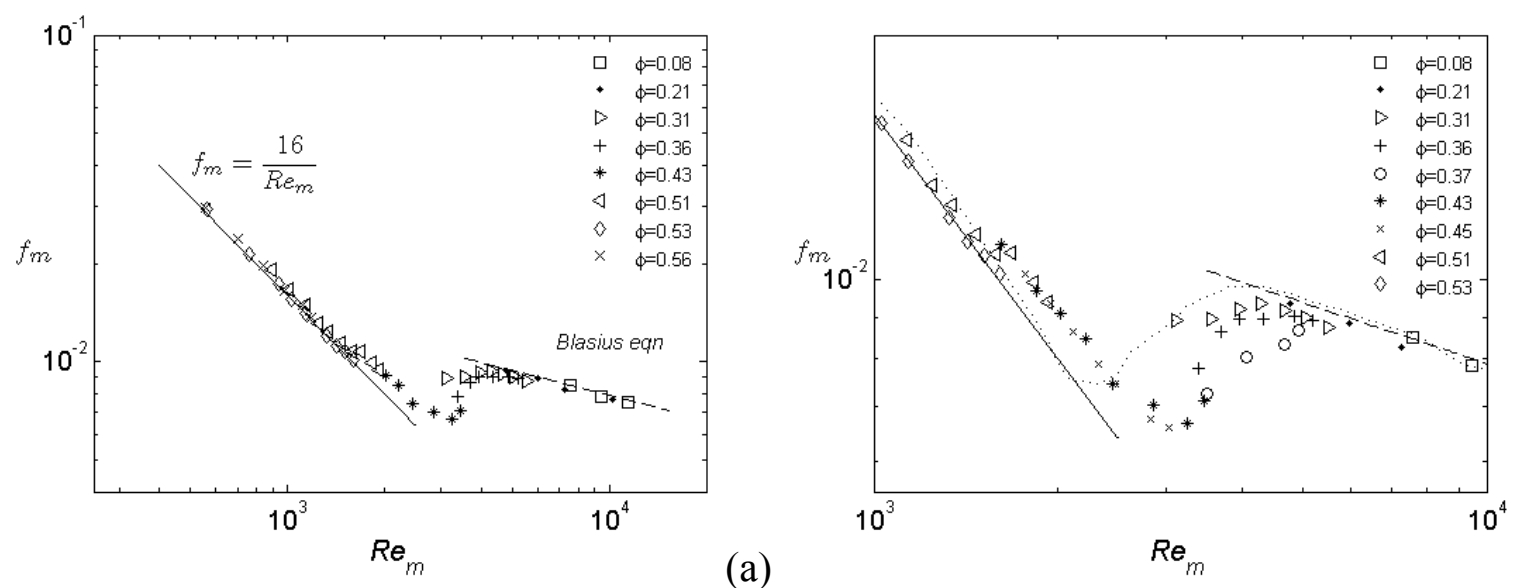

(b)

Figure 15: (a) Wall friction factor as a function of the Reynolds number, (b) Zoom of the transition region including Nikuradse experimental data (...)

\section{Conclusion}

Homogeneously dispersed oil in water pipe flows were studied for a wide range of flow parameters (mixture velocity and phase fraction). The dispersed phase is polydispersed with a mean drop size of about $25 \mu \mathrm{m}$ and is stable during the measurements. Local velocity measurements have been performed at different mixture velocity and concentration, using Particle Image Velocimetry in a matched refractive index medium. The velocity fields measured for a wide range of flow regimes have shown that the emulsion behaves as a Newtonian single phase flow in the turbulent, intermediate and laminar regimes. The effective viscosity deduced from these velocity measurements is in agreement with the evolution of the 
pressure drop as a function of the mixture velocity in the different regimes. The evolution of the viscosity with the concentration of the dispersed phase can be described by both Krieger and Dougherty's and Eilers' models over the whole range of concentration $(0<\phi<0.56)$, setting the maximum concentration to 0.74 . This result validates the Newtonian behaviour of the present emulsion. Using this effective viscosity, the friction factor has been plotted as a function of the mixture Reynolds number. The laminar and turbulent regime trends match Hagen-Poiseuille's and Blasius' laws respectively. These results are in agreement with the earlier work of Baron, ${ }^{16}$ Cengel et al., ${ }^{17}$ Faruqui and Knudsen, ${ }^{19}$ as well as that of $\mathrm{Pal}^{18}$ for stabilized emulsions.

However, above a concentration of 0.31 , a delay to the onset of transition is observed as the concentration is increased ( $R e_{\text {crit }} \sim 3000$ for $\phi=0.45$ ), as well as the width of this regime in terms of Reynolds number. Such flow behaviour is in agreement with the observations of Matas et al. $^{21}$ Further experimental and/or numerical investigations are required in the transition regime to validate this trend.

\section{References}

1. Derkach, S.R. Rheology of emulsion. Advances in Colloid and Interface Science. 2009; 151:1-23

2. Arirachakaran S., Oglesby K.D., Malinovski M.S., Shoham O., Brill JP. An analysis of oil/water flow phenomena in horizontal pipes. SPE Production Operating Symp. Oklahoma, March 13-14, 1989; $155-167$

3. Nädler N., Mewes D. Flow induced emulsification in the flow of two immiscible liquids in horizontal pipes. Int. J. Multiphase Flow. 1997; 23:55-68

4. Angeli P., Hewitt G.F. Flow structure in horizontal oil-water flow. Int. J. Multiphase Flow. 2000; 26: $1117-1140$

5. Elseth G. An Experimental Study of Oil/Water Flow in Horizontal Pipes. Thesis NTNU. Norway 2001

6. Tsuji Y., Morikawa Y., Shiomi H. LDV measurements of an air-solid two-phase flow in a vertical pipe. J. Fluid Mech. 1984;139:417-434

7. Lance M., Bataille J. Turbulence in the liquid phase of a uniform bubbly air-water flow. J. Fluid Mech. 1991; 222:95-118

8. Kulick J.D., Fessler J.R., Eaton J.K. Particle response and turbulence modification in fully developed channel flows. J. Fluid Mech. 1994; 277:109-134

9. Yarin LP, Hetsroni G. Turbulence intensity in dilute two-phase flows. 3. The particle-turbulence interaction in dilute two-phase flows. Int. J. Multiphase Flow. 1994; 20(1):27-44 
10. Suzuki Y., Ikenoya M., Kasagi N. Simultaneous measurement of fluid and dispersed phases in a particle-laden turbulent channel flow with the aid of 3-D PTV. Exp. in Fluids. 2000; 29(suppl.):185193

11. Hosokawa S., Tomiyama A. Turbulence modification in gas-liquid and solid-liquid dispersed two-phase pipe flows. Heat \& Fluid Flow. 2004; 25:489-498

12. Kitagawa A., Hishida K., Kodama Y. Flow structure of microbubble-laden turbulent channel flow measured by PIV combined with the shadow image technique. Exp. in Fluids. 2005; 38: 466-475

13. Kvandal H. K., Elseth G., Melaaen M.C. Measurement of velocity and phase fraction in dispersed two-phase flow. Int. Symp. on Multiph. Flow and Transport Phenom. Antalya, Turkey. 2000; 333-340

14. Hu B., Matar O.K., Hewitt G.F., Angeli P. Mean and turbulent fluctuating velocities in oil-water vertical dispersed flows. Chem Eng Sci. 2007; 62:1199-1214

15. Sundaresan S., Eaton J., Koch D.L., Ottino J.M. Appendix 2: Report of study group on dispersed flow. Int. J. Multiphase Flow. 2003; 29:1069-1087

16. Baron T., Sterling C.S., Schueler A.P. Viscosity of suspensions-Review and application to twophase flow, Proc. Midwest Conf. Fluid Mech. 1953; 3:103

17. Cengel J.A. Faruqui A., Finnigan J.W., Wright C.H., Knudsen J.G. Laminar and turbulent of unstable liquid-liquid emulsions. AIChE J. 1962; 8:335-339

18. Pal R. Pipeline flow of unstable and surfactant-stabilized emulsions, AIChE J. 1993; 39:1754-1764

19. Faruqui A.A., Knudsen J.G. Velocity and temperature profiles of unstable liquid-liquid dispersions in vertical turbulent flow. Chem Eng Sci. 1962; 17:897-907

20. Ward J.P., Knudsen J.G. Turbulent Flow of Unstable Liquid-Liquid Dispersions: Drop Sizes and Velocity Distribution, AIChE J. 1967;13:356-365

21. Matas J.-P., Morris J.F., Guazzelli E. Transition to turbulence in Particulate Pipe Flow. Physical Review Letters. 2003; 90:1

22. Conan C., Masbernat O., Décarre S, Liné A. Local hydrodynamics in a dispersed-stratified liquidliquid pipe flow. AIChE J. 2007; 53:2754-2768

23. Christensen K. T. The influence of peak-locking errors on turbulence statistics computed from PIV ensembles. Exp. in Fluids. 2004;36:484-497

24. Abbas M., Climent E., Simonin O. Fully coupled simulations of non-colloidal monodispersed sheared suspensions. Trans IChemE Part A. Chem Eng Res \& Des. 2007; 85:778-791

25. Schlichting H., Gersten K. Boundary Layer Theory. Springer 2000

26. Nikuradse J. Laws of Flow in Rough Pipes. NACA TM. 1950; 1292

27. Vand V., Viscosity of solutions and suspensions I. Theory. J. Phys. Colloid Chem. 1948; 52: $277-$ 299

28. Manley R., Mason S.G. Particle motion in sheared suspensions. II. Collisions of uniform spheres. Journal of Colloid Science. 1952; 7:354-369 
29. Krieger I.M., Dougherty T.J. A mechanism for non-Newtonian flow in suspensions of rigid spheres. Trans. Soc. Rheol. 1959; III:137-152

30. Batchelor G.K. The effect of Brownian motion on the bulk stress in a suspension of spherical particles. J. Fluid Mech.1977; 83:97-117

31. Jansen K.M.B., Agterof W.G.M., Mellema J. Droplet breakup in concentrated emulsions. J. Rheol. $2001 ; 45: 227-236$

32. Pal R. Novel viscosity equations for emulsions of two immiscible liquids. J. Rheol. 2001; 45:509520

33. Greene G.A., Irvine T.F., Gyves Jr.T., Smith T. Drag relationships for liquid droplets settling in a continuous liquid. AIChE J. 1993; 39:37-41

34. Eilers H. Die Viskosität von Emulsionen hochviskoser Stoffe als Funktion der Konzentration. Kolloid-Z. 1941; 97:313

35. Pal R. Shear viscosity behavior of Emulsions of Two Immiscible Liquids. J. of Colloid Interface Science. 2000; 225:359-366

36. Climent E., Maxey M.R. Numerical simulations of random suspensions at finite Reynolds numbers. Int. J. Multiphase Flow. 2003; 29:579-601 